\title{
Discovery of carbon-rich Miras in the Galactic bulge
}

\author{
Noriyuki Matsunaga $^{1 \star}$, John W. Menzies ${ }^{2}$, Michael W. Feast ${ }^{3,2}$, \\ Patricia A. Whitelock ${ }^{2,3}$, Hiroki Onozato ${ }^{4}$, Sudhanshu Barway ${ }^{2}$, \\ and Elias Aydi 2,3 \\ 1 Department of Astronomy, The University of Tokyo, 7-3-1 Hongo, Bunkyo-ku, Tokyo 113-0033, Japan \\ 2 South African Astronomical Observatory, PO Box 9, Observatory 7935, South Africa \\ 3 Astronomy Department, University of Cape Town, Rondebosch 7701, South Africa \\ 4 Astronomical Institute, Graduate School of Science, Tohoku University, 6-3 Aramaki Aoba, Aoba-ku, Sendai, Miyagi 980-0857, Japan
}

Accepted 2017 May 15. Received 2017 May 15; in original form 2017 Feb 28

\begin{abstract}
Only one carbon-rich (C-rich, hereinafter) Mira variable has so far been suggested as a member of the Galactic bulge and this is in a symbiotic system. Here we describe a method for selecting C-rich candidates from an infrared colour-colour diagram, $\left(J-K_{\mathrm{s}}\right)$ vs ([9] - [18]). Follow-up low-resolution spectroscopy resulted in the detection of $8 \mathrm{C}$ rich Mira variables from a sample of 36 candidates towards the Galactic bulge. Our near-infrared photometry indicates that two of these, including the known symbiotic, are closer than the main body of the bulge while a third is a known foreground object. Of the 5 bulge members, one shows He I and [O II] emission and is possibly another symbiotic star. Our method is useful for identifying rare C-rich stars in the Galactic bulge and elsewhere. The age of these C-rich stars and the evolutionary process which produced them remain uncertain. They could be old and the products of either binary mass transfer or mergers, i.e. the descendants of blue stragglers, but we cannot rule out the possibility that they belong to a small in-situ population of metal-poor intermediate age $(<5$ Gyr $)$ stars in the bulge or that they have been accreted from a dwarf galaxy.
\end{abstract}

Key words: Galaxy: bulge - stars: carbon

\section{INTRODUCTION}

Work in recent years on the Galactic bulge has shown that it is complex, consisting of stars with a range of chemical compositions, ages and distributions (e.g., Ness \& Freeman 2016; McWilliam 2016; Catchpole et al. 2016). It is striking that although Mira variables are common in the bulge only one carbon-rich Mira has been suggested as a possible bulge member, and that is in a symbiotic system (Miszalski, Mikołajewska, \& Udalski 2013). However, a small number of giant carbon-rich stars have been found in the Galactic bulge (Azzopardi et al. 1991; Tyson \& Rich 1991) and their nature will be considered below.

Miras are long period ( $P \geq 100$ days), large amplitude variables lying at the top of the asymptotic giant branch (AGB). Kinematic and other studies (Feast \& Whitelock 2000; Feast 2008; Menzies et al. 2011) indicate that the Mira pulsation period is a good indicator of age and initial mass, and theoretical evolutionary models of single

^ E-mail:matsunaga@astron.s.u-tokyo.ac.jp low to intermediate mass stars are consistent with this (Vassiliadis \& Wood 1993). Miras are separated into two major groups according to their surface chemistry: oxygenrich and carbon-rich (hereinafter, O-rich and C-rich, respectively). In addition to $\mathrm{CO}$ molecules which are present in the atmospheres of stars in both groups, O-rich stars, which have $\mathrm{C} / \mathrm{O}<1$, show molecules such as $\mathrm{H}_{2} \mathrm{O}$, $\mathrm{TiO}$ and $\mathrm{SiO}$ whereas C-rich stars, with $\mathrm{C} / \mathrm{O}>1$, show molecules such as $\mathrm{C}_{2}$ and $\mathrm{CN}$. Stars born from material of normal composition have more oxygen than carbon, but thermal pulses in shell-burning AGB stars can dredge up carbon produced in the interior to the surface, thereby making them C-rich. The ages and initial metallicities of stars will determine whether they become C-rich or not, and this third dredgeup path will produce C-rich stars aged at around 0.5-5 Gyr (Mouhcine \& Lançon 2003; Marigo et al. 2008).

As regards C-rich stars generally, several other methods of production are possible. The giant and subgiant C-rich $\mathrm{CH}$ stars and Barium stars are in long period binary systems and are believed to have atmospheres polluted by a companion which has passed through the 
AGB phase (McClure \& Woodsworth 1990, and references therein). Cool dwarf C-rich stars have been found in significant numbers. Although they are not all now in binary systems, they must have been paired with a much more massive star at some stage in their evolution (Green \& Margon 1994; Totten, Irwin, \& Whitelock 2000; Green 2013; Plant et al. 2016). Various paths for the formation of CEMP stars (carbon-enriched metal poor stars) are discussed by Beers \& Christlieb (2005). Binary interaction has been suggested for $\mathrm{R}$ type stars (Izzard, Jeffery, \& Lattanzio 2007) and for the Azzopardi bulge C-rich stars (Azzopardi, Lequeux, \& Rebeirot 1988; Whitelcok 1993; Ng 1997), while a binary merge has been suggested for a C-rich Mira in a globular cluster (Feast, Menzies, \& Whitelock 2013).

Cole \& Weinberg (2002) assumed that stars in the bulge with $\left(J-K_{\mathrm{S}}\right)_{0}>2$ were C-rich. However, because mass-losing O-rich AGB stars can also be very red (e.g., Wood, Habing, \& McGregor 1998; Ojha et al. 2007), particularly in metal-rich environments, this is clearly not a sufficient condition for identifying C-rich stars in the bulge (Uttenthaler et al. 2015; Catchpole et al. 2016). The present paper reports the discovery of C-rich Miras in the bulge based on a new method using infrared colours to select candidates and also on follow-up spectroscopy. We also briefly discuss the nature of these stars and their possible relationship to the Azzopardi C-rich stars.

\section{SPECTROSCOPIC OBSERVATIONS}

\subsection{Target selection}

We use the $\left(J-K_{\mathrm{S}}\right)$ and ([9] - [18]) colours to identify candidate C-rich stars (Ishihara et al. 2010). C-rich and O-rich stars, especially those with thick circumstellar dust shells, tend to show different colour trends in these bands. As summarised by Ita et al. (2010), dusty C-rich stars often show the $\mathrm{SiC}$ emission band at $11.3 \mu \mathrm{m}$ within the [9] band in addition to a continuum excess due to amorphous carbon dust, while dusty O-rich stars have the silicate bands at 9.8 and $18 \mu \mathrm{m}$ (Lançon \& Wood 2000; Loidl, Lançon, \& Jørgensen 2001; Wright et al. 2009). In the near-infrared bands, C-rich AGB stars have strong $\mathrm{C}_{2}$ and $\mathrm{CN}$ bands in $J$, whilst there are no correspondingly strong bands in $K_{\mathrm{S}}$ which, unlike the $K$ band, is minimally affected by the strong CO band longward of $2.29 \mu \mathrm{m}$. In contrast, for O-rich AGB stars, strong $\mathrm{H}_{2} \mathrm{O}$ absorption has a large impact on both $J$ and $K_{\mathrm{S}}$ magnitudes while $\mathrm{TiO}$ and $\mathrm{VO}$ bands affect $J$. These features at least give a qualitative explanation for the different locations of C-rich and O-rich Miras.

As the primary list of Miras towards the bulge we used the catalogue by Soszyński et al. (2013) which contains 6528 Miras, together with more than 230,000 other types of longperiod variable as a result of the third phase of Optical Gravitational Lensing Experiment (OGLE). The near- and midinfrared colours are taken from the 2MASS (Skrutskie et al. 2006) and AKARI (Ishihara et al. 2010) surveys, respectively. Among the OGLE-III Miras, we found that 4148 objects have both of the required colours, and we identified 66 candidates that fall in the region expected for C-rich stars (Fig. 1, region E). We observed 33 of them, listed in Table 1, as we describe below.

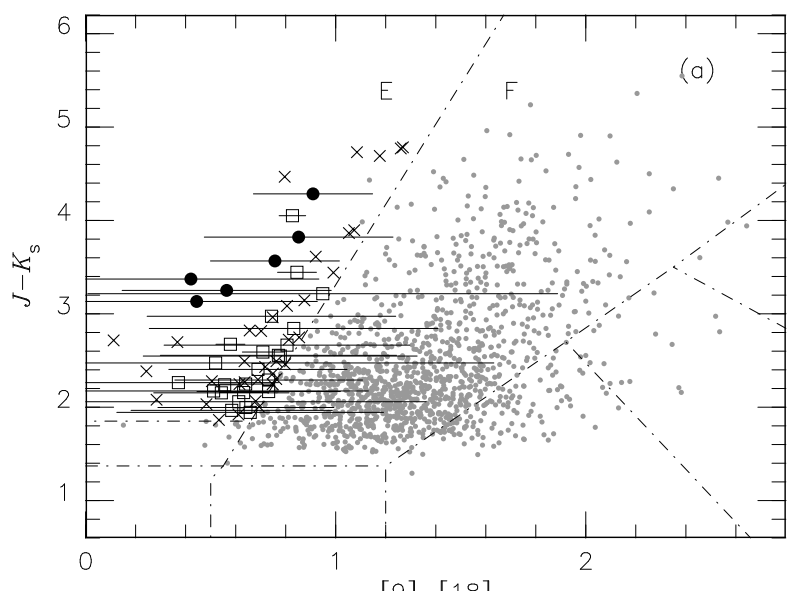

$[9]-[18]$

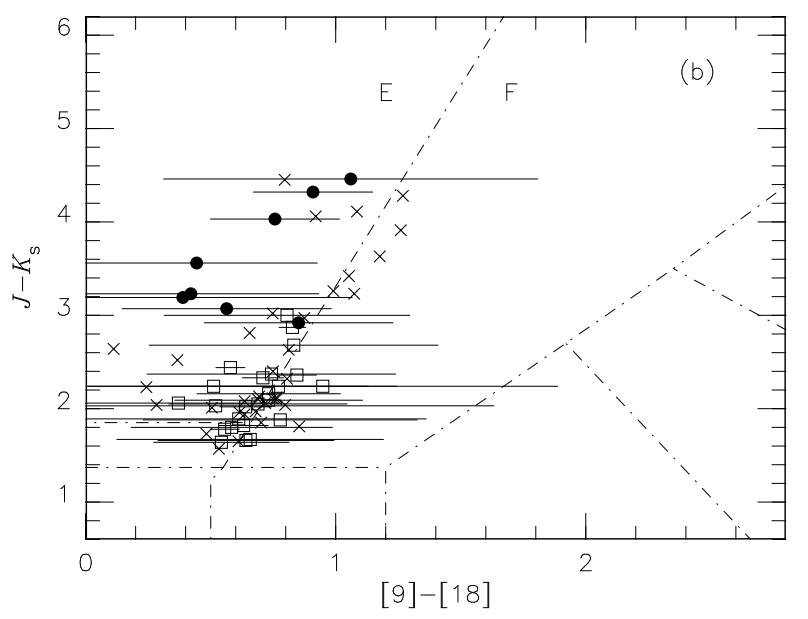

Figure 1. Colour-colour diagram, $\left(J-K_{\mathrm{S}}\right)$ vs ([9] - [18]), for OGLE-III Miras in the bulge indicated by grey dots and for our spectroscopic targets. Those found to be C-rich and O-rich are indicated by filled circles and open squares, respectively, while crosses indicate those that were not observed or were unclassified. Error bars for [9] - [18] colours are given for targets that we describe in this work. Panels (a) and (b) use 2MASS and IRSF near-infrared colours, respectively, except for three objects whose $J-K$ colours are taken from Catchpole et al. (2016) in panel (b). The regions $\mathrm{E}$ and $\mathrm{F}$ are defined by Ishihara et al. (2011) and host mainly C-rich and O-rich stars, respectively.

We added a few targets from the catalogue of Catchpole et al. (2016), which is based on JHKL photometry (our numbers 06, 07, and 33). We combined their $J-K$ colours (without system transformation) with the AKARI [9] - [18] colours from the catalog by Ishihara et al. (2010) for identifying them as C-rich candidates. Object No. 06 (IRAS 17446-4048) was already identified as C-rich based on its IRAS/LRS spectrum (Groenewegen, de Jong, \& Baas 1993); it is also listed in the General Catalog of Galactic Carbon stars (Alksnis et al. 2001).

When we cross correlate the variable catalogues (Soszyński et al. 2013; Catchpole et al. 2016) with the photometric ones (2MASS and $A K A R I$ ), we used a relatively large radius, $5^{\prime \prime}$, in order to avoid missing any C-rich stars whose positions got somehow disturbed by error. Nevertheless, every target we observed has good positional matches in the 2MASS and AKARI catalogues within 2.5" (mostly 
within $0.5^{\prime \prime}$ of a 2 MASS counterpart and within $1.5^{\prime \prime}$ of an $A K A R I$ counterpart). Miras almost always dominate other nearby stars in the infrared and the cross-correlation with infrared catalogues is straightforward.

It should be noted that the interstellar reddening can affect our target selection. Although the OGLE-III survey regions (selected for their $I$-band survey) are expected to be less affected by the interstellar reddening than the Galactic mid-plane, in some regions $A_{V}$ reaches $6 \mathrm{mag}$ (see e.g. Nataf et al. 2016b), which corresponds to $\sim 0.8 \mathrm{mag}$ in $E_{J-K_{\mathrm{s}}}$. Considering that the effect of the reddening is larger in the near infrared than in the mid infrared, this may give offsets on the colour-colour diagram which bring some objects near the boundary from the region $\mathrm{F}$ (for O-rich) to $\mathrm{E}$ (for Crich). We have not corrected the photometry of the selected candidates for interstellar reddening (in Fig. 1) although this might possibly be done in an approximate way using nearby giants. Again, the priority of our target selection was to include as many C-rich candidates as possible.

\subsection{Observations and data analysis}

For this programme we used the SpUpNIC spectrograph (Crause et al. 2016) on the SAAO 1.9-m telescope at Sutherland. Because the Miras are all very red, we installed the GR8 grating with a resolving power of 1200 to cover the wavelength range from 5600 to $9200 \AA$. With a slit width corresponding to 2.1 arcsec, this gave spectra with a resolution of about $8 \AA$. Conditions were rarely photometric throughout the one week's observing run, 13-19 July, 2016, and the Moon was near full, resulting often in high sky backgrounds.

The spectra were bias-corrected and flat-fielded in the usual way, and IRAF routines were used to calibrate the spectra in wavelength. For some objects, close companions coupled with relatively poor seeing made extraction of the target spectra difficult. While the SpUpNIC usually reads out images with 2 pixels binned (in the spatial direction), we observed the object No. 05 without this binning to maximize chance of separating it from a much brighter M-type companion. No attempt has been made to flux-correct the spectra.

\section{$2.3 \quad$ Results}

Spectra for 32 targets (Table 1) were of sufficient quality for further analysis. That for No. 8 is of low quality but a tentative classification seems plausible. A further three targets (Nos. 34, 35, and 36) were either too faint or too badly blended to be useful, so they are not included in the following discussion.

Figs. 2 and 3 show the spectra for C-rich and O-rich Miras respectively. The spectra in Fig. 2 clearly show the sequential vibrational bands of $\mathrm{CN}, 2-0,3-1$, and $4-2$, between 7850 and $8400 \AA$ (Wyckoff 1970). No. 08 has a relatively bright close companion that, together with seeing and bright sky background, made it difficult to extract the spectrum, resulting in a poor signal-to-noise ratio. There is no evidence of $\mathrm{TiO}$ bands and it is probably a C-rich star based on the appearance of the spectrum in the region around the CN bands. The spectra in Fig. 3, in contrast, are characterized by strong TiO bands at 8194 and $8430 \AA$ A together with
Table 1. List of targets we observed with the SpUpNIC spectrograph and their types. The "Object" column gives the OGLE ID numbers in the form of OGLE-BLG-LPVNNNNNN except the three taken from Catchpole et al. (2016) with the IDs of $\mathrm{C} 16 \mathrm{CCN}$.

\begin{tabular}{|c|c|c|c|c|c|c|}
\hline No. & Object & $\begin{array}{l}l \\
\left(^{\circ}\right)\end{array}$ & $\begin{array}{l}b \\
\left(^{\circ}\right)\end{array}$ & $\begin{array}{c}\text { UT } \\
\text { (in 2016) }\end{array}$ & $\begin{array}{l}\operatorname{Exp} \\
(\mathrm{s})\end{array}$ & Type \\
\hline 01 & 149402 & +2.0186 & -2.0559 & $7 / 13$ 19:24 & 420 & $\mathrm{C}^{\dagger}$ \\
\hline 02 & 145820 & -2.7870 & -4.7249 & $7 / 1422: 37$ & 2400 & $\mathrm{C}$ \\
\hline 03 & 056745 & -0.3282 & -1.2751 & $7 / 13$ 22:09 & 600 & $\mathrm{C}$ \\
\hline 04 & 169921 & -0.1137 & -3.7556 & 7/13 21:03 & 600 & $\mathrm{C}$ \\
\hline 05 & 189627 & +2.4710 & -2.9176 & $7 / 1420: 23$ & 1200 & $\mathrm{C}$ \\
\hline 06 & $\mathrm{C} 16 \mathrm{CC} 1$ & -9.9378 & -6.5831 & $7 / 18$ 18:38 & 300 & $\mathrm{C}$ \\
\hline 07 & C16CC3 & +7.3177 & -8.0958 & $7 / 18$ 18:54 & 600 & $\mathrm{C}$ \\
\hline 08 & 230835 & +7.1745 & -4.7850 & $7 / 1701: 07$ & 1200 & C: \\
\hline 09 & 194902 & +2.2482 & -3.2756 & $7 / 1500: 39$ & 300 & M \\
\hline 10 & 013404 & +0.1418 & 2.5743 & $7 / 13$ 20:17 & 420 & M \\
\hline 11 & 031232 & +3.9599 & 2.3630 & $7 / 15$ 00:51 & 300 & M \\
\hline 12 & 039024 & +4.7764 & 2.4548 & $7 / 18$ 18:23 & 300 & $\mathrm{M}$ \\
\hline 13 & 039561 & +5.3812 & 2.7934 & $7 / 16$ 19:00 & 300 & M \\
\hline 14 & 110786 & -2.3417 & -3.5774 & $7 / 1320: 40$ & 300 & M \\
\hline 15 & 122034 & -1.8007 & -3.5504 & $7 / 1623: 35$ & 300 & M \\
\hline 16 & 127293 & +0.2527 & -2.5135 & $7 / 17$ 18:55 & 300 & M \\
\hline 17 & 131690 & -0.3688 & -2.9776 & $7 / 1720: 07$ & 300 & $\mathrm{M}$ \\
\hline 18 & 133170 & +0.1915 & -2.6888 & 7/19 00:06 & 300 & M \\
\hline 19 & 170446 & +1.8618 & -2.6492 & $7 / 15 \quad 00: 22$ & 300 & $\mathrm{M}$ \\
\hline 20 & 188857 & +0.0420 & -4.2511 & $7 / 1500: 08$ & 300 & M \\
\hline 21 & 195036 & +1.8849 & -3.4856 & $7 / 1623: 55$ & 300 & $\mathrm{M}$ \\
\hline 22 & 195810 & +2.0728 & -3.4196 & $7 / 1323: 04$ & 600 & M \\
\hline 23 & 214635 & +5.1753 & -2.6767 & $7 / 1701: 45$ & 300 & M \\
\hline 24 & 216952 & -0.1630 & -5.7607 & $7 / 1700: 13$ & 1200 & M \\
\hline 25 & 230250 & +6.9510 & -4.2590 & $7 / 1621: 18$ & 120 & M \\
\hline 26 & 072230 & -0.2513 & -1.5968 & 7/14 18:19 & 900 & M \\
\hline 27 & 076743 & +0.5348 & -1.2234 & $7 / 1321: 38$ & 600 & M \\
\hline 28 & 110674 & -0.9474 & -2.7629 & $7 / 1900: 27$ & 300 & M \\
\hline 29 & 216273 & +2.4655 & -4.2839 & $7 / 1620: 12$ & 600 & $\mathrm{M}$ \\
\hline 30 & 194903 & -0.2322 & -4.6589 & $7 / 1622: 01$ & 600 & $\mathrm{M}$ \\
\hline 31 & 168224 & +0.9524 & -3.1055 & $7 / 17 \quad 20: 23$ & 300 & $\mathrm{M}$ \\
\hline 32 & 132552 & +1.2480 & -2.0630 & $7 / 16 \quad 19: 33$ & 1200 & $\mathrm{M}$ \\
\hline 33 & C16CC5 & +5.7962 & -7.9836 & 7/18 19:16 & 600 & M \\
\hline 34 & 082471 & -0.3249 & -1.8431 & $7 / 1322: 38$ & 600 & - \\
\hline 35 & 151973 & +1.4302 & -2.4562 & $7 / 15$ 01:29 & 360 & - \\
\hline 36 & 179847 & +0.8947 & -3.4740 & $7 / 1323: 28$ & 600 & - \\
\hline
\end{tabular}

$\dagger$ No. 1 is the C-rich star (H1-45) identified by Miszalski et al. (2013) as a symbiotic.

TiO bands longward of $7667 \AA$ beside the telluric A band at around $7594 \AA$ (Wyckoff 1970; Lançon \& Wood 2000). We thus gave the classification as listed in Table 1, seven C-rich, one probably C-rich and 25 O-rich Miras.

In Fig. 2, our spectrum of No. 01 is similar to that shown by Miszalski et al. (2013) who identify the star (H1$45)$ as a symbiotic. The spectrum of No. 02, though rather weak, shows emission lines from $\mathrm{H} \alpha$, He I (7065 $\AA$ ) and [O II] $(7319+7330 \AA)$ as in that of No. 1 . The He and [O II] lines in particular suggest a binary system, but there are no suitable diagnostic high excitation lines in this part of the spectrum to allow us to classify it definitely as symbiotic. The Raman bands at 6825 and $7082 \AA$ are absent from both spectra, but this probably indicates that the companion star does not have a sufficiently high temperature to excite them (see the discussion in Miszalski et al. 2013). 


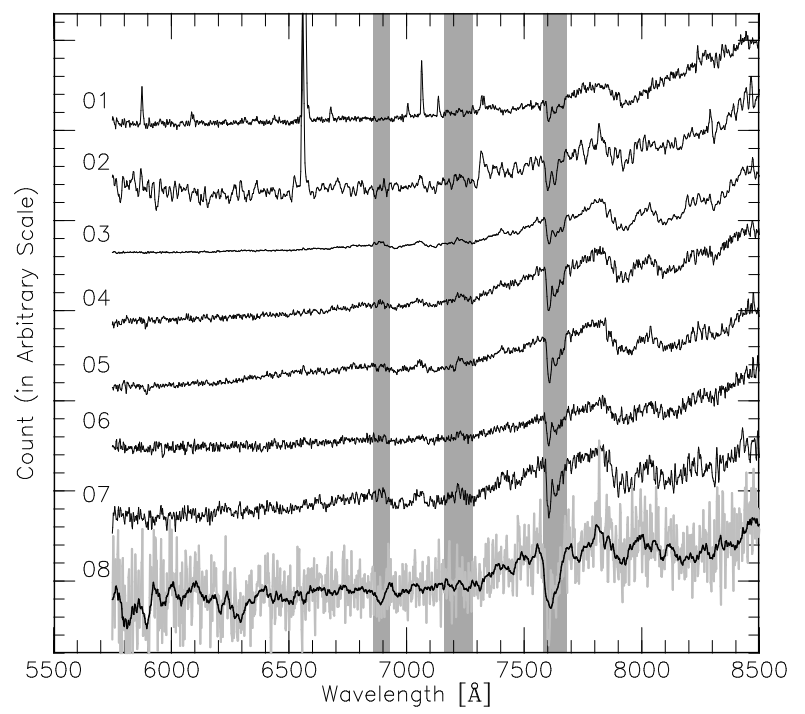

Figure 2. Spectra of C-rich Miras. The telluric absorption features, the strongest of which are due to $\mathrm{O}_{2} 6867 \AA$ (B band), $\mathrm{H}_{2} \mathrm{O}$ $7164 \AA$ and $\mathrm{O}_{2} 7594 \AA$ (A band), are shown as shaded vertical bands. The spectrum of No. 8 has a rather low signal-to-noise ratio as illustrated by the grey curve, but the black curve after smoothing shows the similarity to the other spectra.

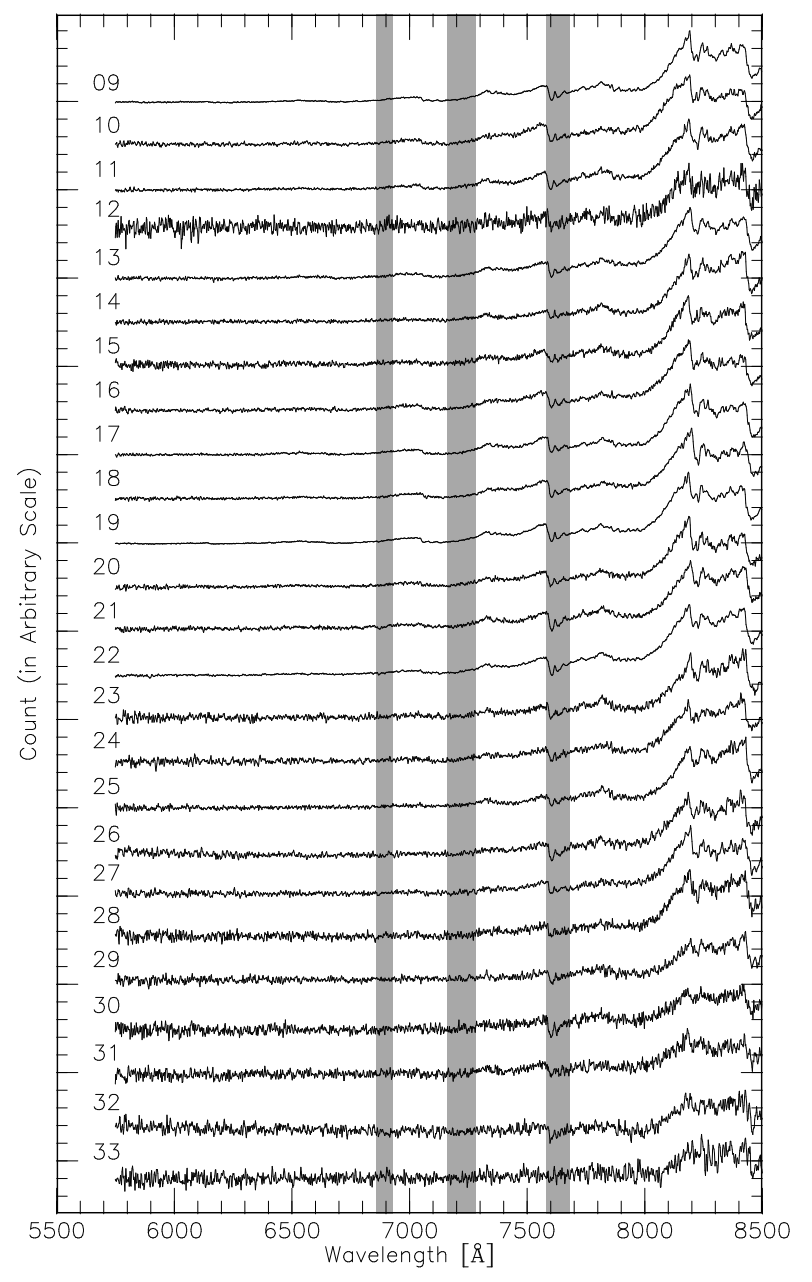

Figure 3. Same as Fig. 2 but for O-rich Miras.

\section{FOLLOW-UP PHOTOMETRY AND DISTANCES}

\subsection{New photometric data}

We used the 1.4-m Infrared Survey Facility at Sutherland, with the SIRIUS near-infrared camera (Nagashima et al. 1999; Nagayama et al. 2003), to estimate mean magnitudes of our targets. We observed each target for 6-9 epochs between 2015 June and 2016 August. For No. 36, we obtained 18 measurements in each band, but we used two different integration times and so there are pairs of measurements close in time at each epoch. Fig. 4 plots phased light curves of C-rich Miras in $J H K_{\mathrm{S}}$ in addition to the ones in the OGLE $I$ band. The third-order Fourier series,

$m(t)=m_{0}+\sum_{i=1}^{3} A_{i} \cos \left(2 \pi i t / P+\phi_{i}\right)$

was fitted for the $I$ band, and it was adjusted to fit the light curves in $J H K_{\mathrm{S}}$ with changing the mean $\left(m_{0}\right)$, total amplitude, and maximum phase. The amplitudes of our C-rich objects are larger than $1 \mathrm{mag}$, which is typical for C-rich Miras (Whitelock et al. 2006). The derived means are compared with the simple averages of $N$ measurements in Table 2. The 2MASS magnitudes, crosses in Fig. 4, were not considered when the amplitudes were scaled and tend to be offset from the fitted curves (see the next paragraph). Although our photometric data show relatively large gaps in phase, the light curves seem to give reasonable estimates of mean. For the six C-rich Miras in Fig. 4, the difference between the two kinds of mean is estimated to be approximately $-0.1( \pm 0.2) \mathrm{mag}$ in each of the $J H K_{\mathrm{S}}$. This suggests that our estimates of mean are as good as 0.2 mag. The mean magnitudes from the fitted light curves are used in the following, but the difference between two kinds of means does not affect the conclusions. For the three objects from Catchpole et al. (2016), we adopt the published JHK magnitudes which are averages of two measurements in most cases.

It is worthwhile to give some further comments on the light curves of our C-rich Miras. The OGLE-III I-band light curves of the C-rich Miras are plotted in Fig. 5 when available. All of them show cycle-to-cycle variations except No. 02 for which the time coverage is very short. It is known that C-rich Miras tend to show such variations more often than O-rich ones (Whitelock et al. 2003, 2006). The fact that the 2MASS points in Fig. 4 are not aligned with the IRSF light curves may be explained by such variations since the 2MASS photometry was obtained over 15 years ago.

For two objects, Nos. 03 and 08, the fitted curves show large offsets in phase between the $I$-band and $J H K_{\mathrm{S}}$-band ones in Fig. 4. The long time interval between the OGLEIII datasets and our photometry, $\sim 10$ years, makes it difficult to discuss such offsets, they can be understood as follows. No. 03 shows a large fluctuation in the $I$-band light curve and some cycles seem to show unphased trends. The OGLEIII light curve of No. 08 covers less than two cycles (Fig. 5) and seems to show a variation between the two minima. The period and phase may have significant errors which produce the inconsistency in phase between the two datasets.

As presented in Fig. 1, all eight C-rich stars fall in the region $\mathrm{E}$ although the large errors in [9] - [18] make it hard 

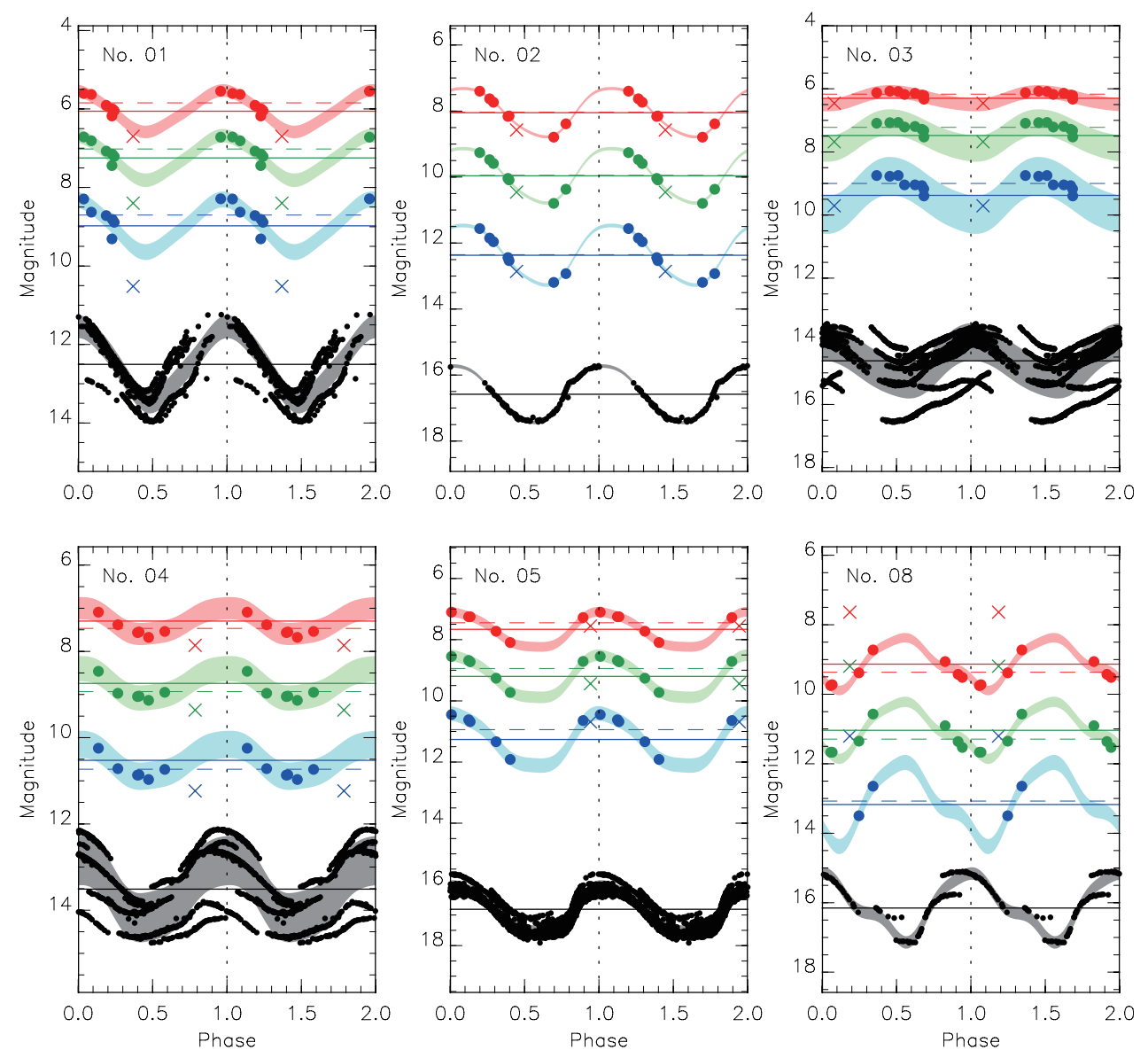

Figure 4. Phased light curves of C-rich Miras. Black, blue, green, and red points indicate photometric measurements in $I, J, H$, and $K_{\mathrm{s}}$. The cross symbols mark 2MASS magnitudes. The fitted light curves are drawn for each band. The width of a $I$-band curve corresponds to the standard deviation of the photometric points around the fitted curve, while the widths of the curves for other bands correspond to that of the $I$ band but scaled according to the difference in amplitudes. The filled and dashed horizontal lines indicate the mean magnitude for a fitted curve and the simple average of the photometric points, respectively.

to be sure of their precise locations in this diagram. On the other hand, many of the candidates are found to be actually O-rich, especially among those near the boundary between the regions E and F (Fig. 1). In fact, our follow-up photometry suggests that there were biases in the 2MASS $J-K_{\mathrm{S}}$ colours that we used for the targets selection. This may be due to colour variations during a cycle; the 2MASS magnitudes were single-epoch values. Some O-rich stars could have been included in our C-rich candidates due to the interstellar reddening as mentioned in Section 2.1.

It should be pointed out that there are clearly O-rich stars with $J-K_{\mathrm{S}}$ colours significantly larger than 2 mag. There are such red stars among our spectroscopic O-rich samples, and a number of objects among the OGLE-III bulge Miras have similarly large $J-K_{\mathrm{S}}$ and are located in the region F. These are probably O-rich objects with thick circumstellar dust shells. The $J-K_{\mathrm{S}}$ colour is not sufficient to select C-rich stars.

\subsection{Colour-magnitude diagram}

Fig. 6 shows the colour-magnitude diagram of our target Miras. OGLE Miras in the LMC (Soszyński et al. 2009) are compared after a vertical offset corresponding to the approximate difference in distance modulus, $\Delta \mu_{0}=4 \mathrm{mag}$, between the bulge and the LMC. The line-of-sight depth of the bulge is much larger than that of the LMC and the colours and magnitudes of the bulge stars are affected by interstellar extinction, while the LMC Miras have small interstellar reddenings. The very bright Mira is No. 06 which was classified as a C-rich star based on its IRAS/LRS spectrum (Groenewegen, de Jong, \& Baas 1993). This is obviously a foreground object. Whitelock et al. (2006) estimated its distance as $1.40 \mathrm{kpc}$ and foreground extinction, $A_{V}=0.80 \mathrm{mag}$. The other C-rich Miras indicated by filled circles are scattered around a sequence roughly expected for such objects at around the distance of the bulge. Two of them look slightly brighter than the other Miras, and one somewhat fainter; we discuss their distances further below.

The C-rich stars from Azzopardi et al. $(1988,1991)$ are also shown in Fig. 6. These are faint for evolved C-rich AGB stars at the distance of the bulge as previously known (e.g. Azzopardi et al. 1988; Whitelcok 1993; Ng 1997). The faint C-rich stars in the Sculptor dwarf spheroidal (Menzies et al. 2011) have $M_{K_{\mathrm{S}}}<-5.1$, which would give $K_{\mathrm{S}}<9.5$ at the distance of the Galactic bulge. All of the bulge Azzopardi 
Table 2. Photometric data for target Miras. When two magnitudes are given for each of the mean magnitudes $(\langle J\rangle,\langle H\rangle$, and $\left.\left\langle K_{\mathrm{S}}\right\rangle\right)$, the first one is obtained with the fitted light curves and the second one with simply averaging the $N$ measureents.

\begin{tabular}{cccccc}
\hline No. & $\begin{array}{c}\text { Period } \\
\text { (days) }\end{array}$ & $N$ & $\begin{array}{c}\langle J\rangle \\
(\mathrm{mag})\end{array}$ & $\begin{array}{c}\langle\mathrm{H}\rangle \\
(\mathrm{mag})\end{array}$ & $\begin{array}{c}\left\langle K_{\mathrm{s}}\right\rangle \\
(\mathrm{mag})\end{array}$ \\
\hline 01 & 416.2 & 8 & $8.70 / 8.98$ & $7.02 / 7.25$ & $5.84 / 6.06$ \\
02 & 600.5 & 7 & $12.35 / 12.37$ & $9.95 / 9.96$ & $8.04 / 8.05$ \\
03 & 373.6 & 8 & $9.00 / 9.37$ & $7.22 / 7.48$ & $6.17 / 6.30$ \\
04 & 449.7 & 6 & $10.73 / 10.53$ & $8.93 / 8.74$ & $7.47 / 7.30$ \\
05 & 509.8 & 6 & $10.94 / 11.22$ & $8.94 / 9.20$ & $7.45 / 7.66$ \\
$06^{\dagger}$ & 421.6 & - & $8.41 /-$ & $5.79 /-$ & $3.95 /-$ \\
$07^{\dagger}$ & 436.9 & - & $10.34 /-$ & $8.49 /-$ & $7.15 /-$ \\
08 & 512.6 & 7 & $13.07 / 13.16$ & $11.29 / 11.04$ & $9.37 / 9.13$ \\
09 & 348.4 & 7 & $8.61 / 8.90$ & $7.54 / 8.03$ & $6.90 / 7.10$ \\
10 & 365.1 & 7 & $8.17 / 8.60$ & $6.98 / 7.57$ & $6.21 / 6.78$ \\
11 & 420.1 & 6 & $8.72 / 8.89$ & $7.25 / 7.35$ & $6.44 / 6.52$ \\
12 & 436.5 & 9 & $8.88 / 9.30$ & $7.51 / 7.86$ & $6.67 / 6.97$ \\
13 & 398.5 & 9 & $8.13 / 8.09$ & $7.17 / 6.98$ & $6.45 / 6.31$ \\
14 & 432.1 & 8 & $7.90 / 7.87$ & $6.38 / 6.40$ & $5.38 / 5.43$ \\
15 & 352.8 & 7 & $9.39 / 9.35$ & $8.05 / 8.05$ & $7.28 / 7.26$ \\
16 & 393.8 & 7 & $8.70 / 8.89$ & $7.48 / 7.67$ & $6.69 / 6.86$ \\
17 & 379.9 & 8 & $7.87 / 7.89$ & $6.85 / 6.90$ & $6.20 / 6.25$ \\
18 & 424.6 & 6 & $8.60 / 8.77$ & $7.48 / 7.67$ & $6.75 / 6.89$ \\
19 & 353.9 & 7 & $7.66 / 8.05$ & $6.71 / 7.07$ & $6.13 / 6.38$ \\
20 & 304.0 & 6 & $8.55 / 8.49$ & $7.50 / 7.48$ & $6.83 / 6.83$ \\
21 & 377.8 & 7 & $8.84 / 9.30$ & $7.71 / 8.05$ & $6.98 / 7.24$ \\
22 & 426.0 & 6 & $8.58 / 8.93$ & $7.36 / 7.62$ & $6.51 / 6.69$ \\
23 & 393.9 & 8 & $9.25 / 9.00$ & $7.88 / 7.78$ & $6.93 / 6.84$ \\
24 & 574.4 & 7 & $10.30 / 10.76$ & $8.81 / 9.18$ & $7.55 / 7.89$ \\
25 & 584.3 & 8 & $8.10 / 8.04$ & $6.69 / 6.63$ & $5.84 / 5.80$ \\
26 & 506.6 & 8 & $10.22 / 11.00$ & $8.65 / 9.42$ & $7.34 / 8.00$ \\
27 & 387.1 & 8 & $8.82 / 8.97$ & $7.56 / 7.79$ & $6.69 / 6.92$ \\
28 & 426.2 & 8 & $9.30 / 9.40$ & $7.92 / 8.01$ & $6.98 / 7.04$ \\
29 & 382.9 & 6 & $9.09 / 8.68$ & $7.76 / 7.42$ & $6.92 / 6.66$ \\
30 & 362.6 & 7 & $10.97 / 10.63$ & $9.70 / 9.39$ & $8.61 / 8.39$ \\
31 & 324.5 & 7 & $8.83 / 9.15$ & $7.73 / 8.02$ & $7.05 / 7.26$ \\
32 & 482.8 & 7 & $10.69 / 11.17$ & $9.21 / 9.59$ & $8.21 / 8.49$ \\
$33^{\dagger}$ & 404.4 & - & $10.56 /-$ & $8.66 /-$ & $7.48 /-$ \\
34 & 434.9 & 7 & $10.18 / 10.47$ & $8.40 / 8.64$ & $7.04 / 7.21$ \\
35 & 415.9 & 8 & $8.56 / 8.73$ & $7.39 / 7.54$ & $6.66 / 6.79$ \\
36 & 486.8 & 18 & $12.28 / 12.46$ & $10.13 / 10.00$ & $8.20 / 8.18$ \\
\hline & & & & &
\end{tabular}

$\dagger$ JHK magnitudes from Catchpole et al. (2016).

stars are fainter than this, so plausibly assuming that they are not all more distant than the bulge, they cannot have been enriched in carbon through dredge up in a normal star. They are also bluer than C-rich Miras in the LMC and the ones newly found in the bulge (note that the $J-K_{\mathrm{S}}$ colours in Fig. 6 are not dereddened). Ng $(1997,1998)$ discussed the possibility that some of these C-rich stars may belong to the Sagittarius dwarf galaxy, but their natures remain unclear.

The star symbol in Fig. 6 indicates the C-rich star found by Hynes et al. (2014). They identified this as the optical counterpart of an X-ray source, CXOGES J173620.2-293338. The position in the colour-magnitude diagram is close to that of the objects of Azzopardi et al. (1991), but bluer and fainter than our C-rich Miras. It shows small-amplitude irregular variations in its OGLE light curve (Udalski et al. 2012). Its nature is unclear but the associa-

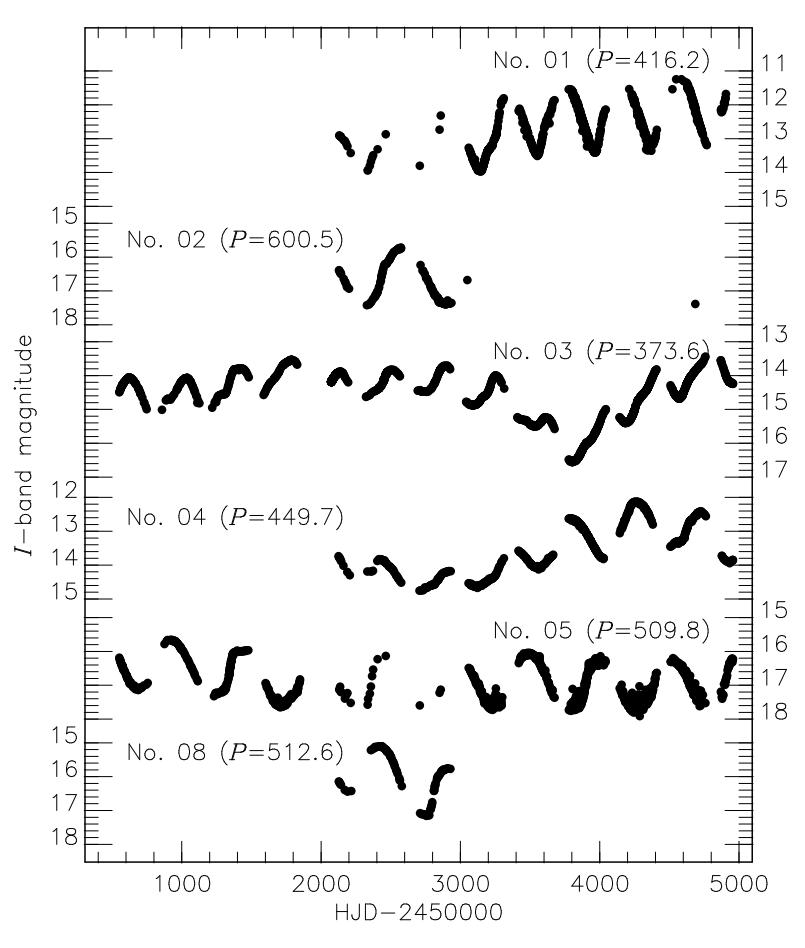

Figure 5. OGLE-III I-band light curves of C-rich Miras.

tion of X-ray emission makes it particularly interesting and suggests that it is a binary source.

In Fig. 6, the O-rich Miras we observed are indicated by open squares, and most of them are significantly bluer than the C-rich targets. This suggests that the latter have intrinsically larger $J-K_{\mathrm{S}}$ as expected. Compared to the Orich Miras in the LMC (grey dots in Fig. 6), our sample of O-rich Miras are redder, presumably due to the combined effect of circumstellar and interstellar dust, and at around the bright end of the LMC distribution. Fig. 7 shows that the spectroscopic targets we selected are biased towards longer periods which explains the higher luminosities.

\subsection{Distances}

Estimating accurate distances to the C-rich Miras is not straightforward. It is known that reddish C-rich Miras appear fainter than predicted by the near-infrared periodluminosity relation for bluer Miras. The deviation from the relation is correlated with the colour in a quadratic manner (Ita \& Matsunaga 2011); such a correlation can be used for correcting the luminosities and estimating the distances. However, Miras towards the bulge are also reddened by interstellar dust. The interstellar extinction is represented by the total-to-selective extinction ratio, $A_{K_{\mathrm{s}}} / E_{J-K_{\mathrm{s}}}=$ 0.494 (Nishiyama et al. 2006), which is different from the quadratic form of the deviation from the period-luminosity relation. These two effects are mixed and difficult to separate.

Here we consider the Wesenheit index, $W_{J K_{\mathrm{S}}}=K_{\mathrm{S}}-$ $0.5\left(J-K_{\mathrm{S}}\right)$, to make approximate corrections for the reddening and dimming. The coefficient 0.5 is close to the totalto-selective extinction ratio and, to some extent, the ratio between the colour and the deviation from the periodluminosity relation, and thus $W_{J K_{\mathrm{s}}}$ is insensitive to both 


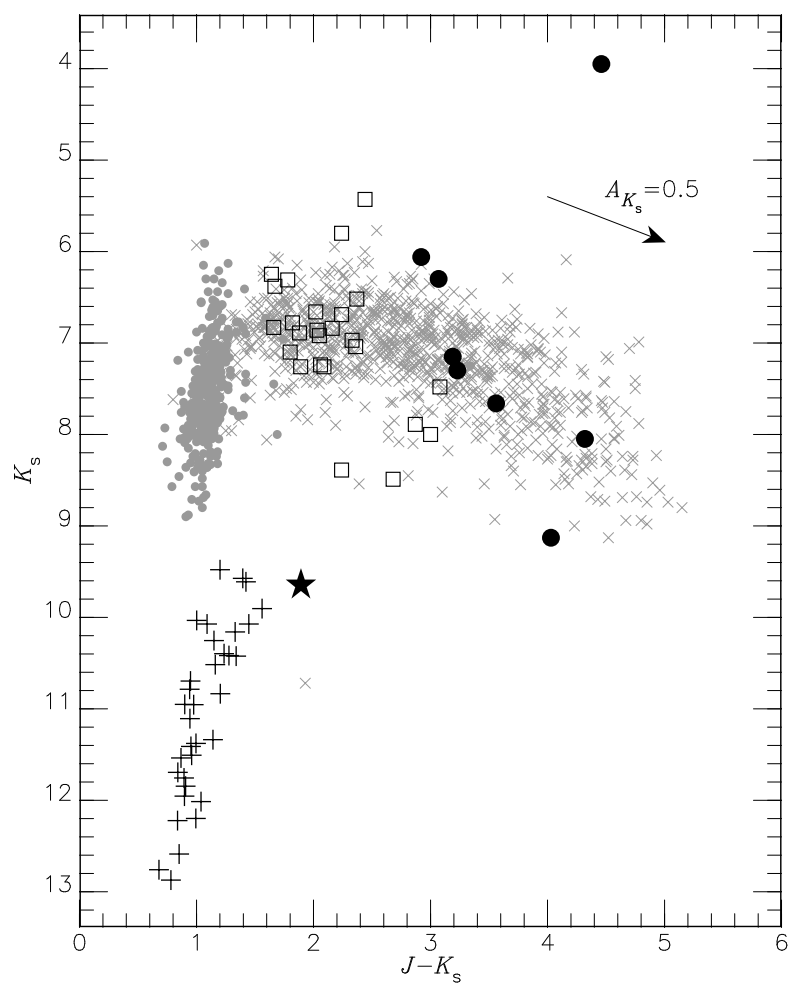

Figure 6. Colour-magnitude diagram. Filled circles indicate C-rich Miras among our targets while we found that the Miras indicated by open squares are O-rich. The plus and star symbols illustrate 2MASS photometry for C-rich stars reported by Azzopardi et al. (1991) and by Hynes et al. (2014), respectively. Grey dots and crosses indicate OGLE-III Miras in the LMC which are classified as O-rich and C-rich, respectively, by Soszyński et al. (2009); their 2MASS $K_{\mathrm{S}}$ magnitudes are offset by $4 \mathrm{mag}$ correcting for the distance difference between the bulge and the LMC.

reddening effects. Fig. 8 plots the same objects, except nonMiras as in Fig. 6 on the $\log P-W_{J} K_{\mathrm{s}}$ diagram. Besides the clearly foreground object, No. 06, two stars (Nos. 01 and 03) are distinctly brighter than the others. They are also bright in Fig. 6. This suggests that the latter two are also foreground objects. Although it is difficult to estimate their distances as mentioned above, they can be located at $\sim 5 \mathrm{kpc}$ from the Sun considering that they look brighter than the bulk of other Miras by approximately $1 \mathrm{mag}$. This is around the short end of the distance estimated by Miszalski et al. (2013) for the symbiotic star No. 01. Those authors also discussed the star's possible membership of the extended bar-like Galactic bulge, which we cannot rule out. The fact that star No. 08 appears to be more distant than the bulk of the bulge stars may be an indication that we are simply observing the large spread of the bulge in the line of sight or it may indicate that this star is undergoing an obscuration event of the type common in C-rich Miras (Whitelock et al. 2006). Future tests of bulge membership should be based on kinematic measurements, both radial velocities from infrared high-resolution spectroscopy and proper motion, e.g. based on Gaia and/or the VVV (VISTA Variables in the Via Lactea) survey (Libralato et al. 2015).

The colours and magnitudes of the other four C-rich Miras, Nos. 02, 04, 05 and 07, are consistent with what are ex-
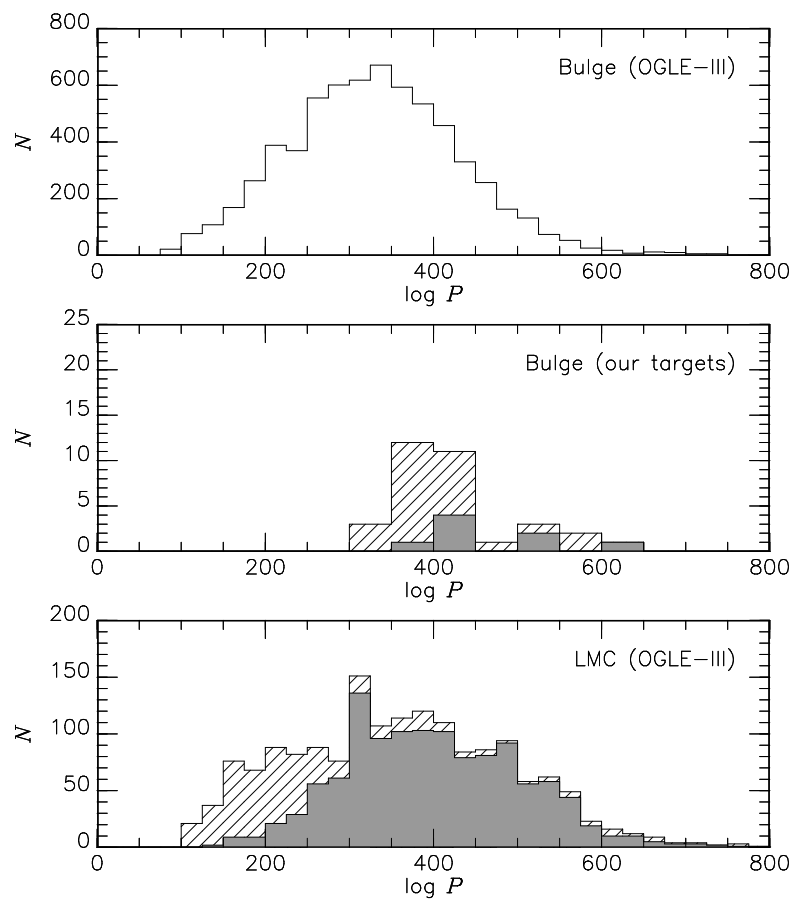

Figure 7. Period distributions of OGLE-III bulge Miras (Soszyński et al. 2013) (top), our targets (middle), and OGLEIII Miras in the LMC (Soszyński et al. 2009) (bottom). Except in the top panel, the filled and shaded portions indicate $\mathrm{C}$ - and O-rich Miras respectively.

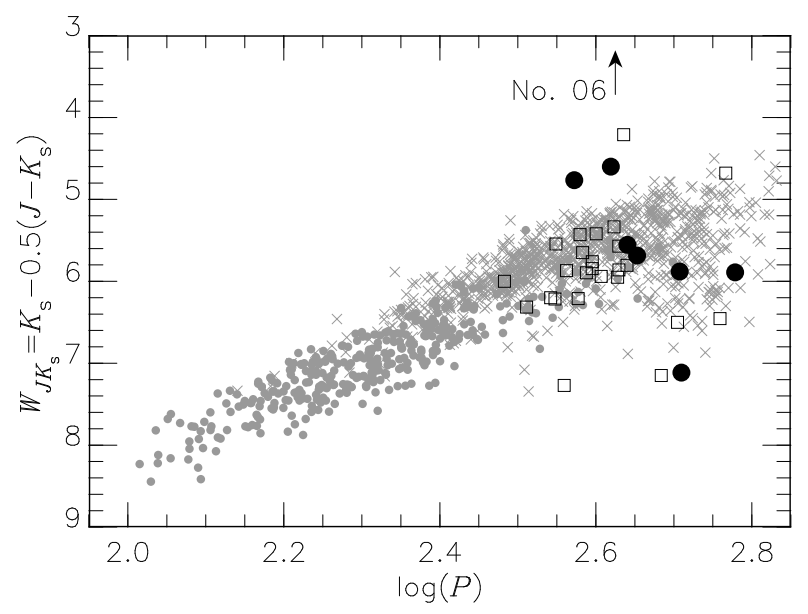

Figure 8. Period-Wesenheit relation. Symbols are the same as in Fig. 6. The bright foreground C-rich Mira, No. 06, is outside the range of the plot but indicated by the arrow.

pected for C-rich Miras at the distance of the bulge (Figs. 6 and 8).

\section{DISCUSSION}

\subsection{Diagnostic to select C-rich Miras}

We have demonstrated that the $\left(J-K_{\mathrm{S}}\right)$ vs ([9] - [18]) diagram effectively isolates candidate $\mathrm{C}$-rich stars, although the separation between C-rich and O-rich stars in that diagram is not definitive. Colour variations and/or photometric 
errors can increase the number of false positive candidates as discussed in Section 3. In a stellar system like the Galactic bulge where O-rich objects are dominant, it is hard to isolate individual C-rich objects. Nevertheless, this method has proved useful enough to find the new C-rich Miras in the bulge. As discussed in Section 3.1, C-rich Miras tend to show cycle-to-cycle variations (Fig. 5). The combination of light curve behaviour and the position in the $\left(J-K_{\mathrm{S}}\right)-([9]-[18])$ diagram, or just the light curve behaviour alone, might be a good diagnostic for selecting candidate C-rich Miras.

\subsection{The origin of C-rich Miras in the bulge}

The origin of the bulge C-rich Miras is difficult to establish. We consider three possibilities below.

The five C-rich Miras (Nos. 03-05, 07, and 08) are, within the uncertainties, in the bulge. They have periods between 373 and 512 days, and are not obviously interacting binaries. If they have evolved from isolated stars they are of intermediate age, 0.5-3 Gyr, according to the correlation between age and period for C-rich Miras (Feast, Whitelock, \& Menzies 2006). This would imply the existence of a small population of intermediate age stars with low metallicities, or at least low oxygen abundances. The presence of intermediate age stars in the bulge has long been suspected, e.g. from the presence there of longer period O-rich Miras and related objects (see Catchpole et al. 2016; Nataf 2016a for reviews, and among others on this general topic, van Loon et al. 2003; Groenewegen \& Blommaert 2005). However, at a time when a case was being made for an entirely old bulge, it was suggested that these variables could be merged binaries (Renzini \& Greggio 1990). More recently evidence has been found for relatively metal-rich dwarfs with ages of $~ 5$ Gyr or younger (Bensby et al. 2013; Haywood et al. 2016). and for stars (of unknown age) with $\alpha$-element abundances below the general bulge $\alpha$-Fe correlation (Recio-Blanco et al. 2017). This serves to illustrate the complexity of the bulge population and further exploration of this point is beyond the scope of this work. If our C-rich Miras are intermediate age stars, this would be the first demonstration that a (small) intermidiate age population exists in the bulge with the correct mass, metallicity, and oxygen abundances to produce $\mathrm{C}$-rich stars in the AGB dredge-up process.

However, older stars can produce more massive, potentially C-rich, progeny through either a mass transfer or a merger process. The C-rich Mira in the globular cluster Lyngå 7 (Feast et al. 2013), with a pulsation period of 551 days, is an example of a merger; the authors argued that the current mass of that Mira was about twice what was expected for a single Mira in that cluster. Such stars could be the descendants of blue stragglers, several of which have been clearly identified in the bulge (Clarkson et al. 2011). It may also be that the Azzopardi C-rich stars are the giant branch and/or AGB predecessors of the C-rich Miras.

The two Miras likely in binary systems (Nos. 01 and 02, considering their symbiotic nature) also have long periods, 416 and 600 days, suggesting that they are binary mergers, i.e. these were once triple systems, or that they have accreted most of the mass of their companions. It is beyond the scope of this paper to discuss the theoretical implications of this in terms of stellar evolution or dynamics. It is certainly no- table that two out of the six C-rich Miras that are in or very near the bulge are binary. Furthermore, the demonstration by Pietrzyński et al. (2012), that a bulge binary system involved an RR Lyr-like star with a mass of only $0.26 \mathrm{M}_{\odot}$, shows that extreme mass transfer events, of the type that would be required to produce long period C-rich Miras, do occur.

A third alternative is that these C-rich Miras have been accreted from a merging dwarf galaxy with a composition similar to that of the Sagittarius dwarf galaxy, which contains numerous C-rich Miras in its core and in its tidal stream (Whitelock, Irwin, \& Catchpole 1996; Law \& Majewski 2016).

The merged binary scenario, or at least the scenario in which one star accretes most of the mass of a companion, is marginally preferred for the origin of these Miras, simply because mass-transfer binaries are found in the bulge and quite possibly become C-rich stars on the AGB. However, in a very large and heterogeneous population such as the bulge it is impossible to be definitive about the origins of a small group of stars. Identification of more C-rich stars and studies of their kinematics and distribution will shed light on the problem. An abundances analysis would help a great deal, but that is not practical for these very cool stars with extended and dynamic atmospheres.

\section{ACKNOWLEDGMENTS}

We are grateful for Lisa Crause's assistance with the new SpUpNIC spectrograph on the first night of our observing run. We thank Shazrene Mohamed for helpful discussions about binary and multiple star systems. This work has been supported as a Joint Research Project under agreement between the the Japan Society for the Promotion of Science (JSPS) and National Research Foundation in South Africa. NM is grateful to Grant-in-Aid (No. 26287028) from the JSPS. MWF, JWM, PAW, and EA acknowledge support from the NRF. The IRSF project is a collaboration between Nagoya University and the South African Astronomical Observatory (SAAO) supported by the Grants-in-Aid for Scientific Research on Priority Areas (A) (Nos. 10147207 and 10147214) and Optical \& Near-Infrared Astronomy InterUniversity Cooperation Program, from the Ministry of Education, Culture, Sports, Science and Technology (MEXT) of Japan and the National Research Foundation (NRF) of South Africa.

\section{REFERENCES}

Alksnis A., Balklavs A., Dzervitis U., Eglitis I., Paupers O., Pundure I., 2001, Baltic Astron. 10, 1

Azzopardi M., Lequeux J., Rebeirot E., 1988, A\&A, 202, L27

Azzopardi M., Lequeux J., Rebeirot E., Westerlund B. E., 1991, A\&AS, 88, 265

Beers T. C., Christlieb N., 2005, ARA\&A, 43, 531

Bensby T. et al., 2013, A\&A, 549, A147

Catchpole R. M., Whitelock P. A., Feast M. W., Hughes S. M. G., Irwin M., Alard C., 2016, MNRAS, 455, 2216

Clarkson W. I. et al., 2011, ApJ, 735, 37

Cole A. A., Weinberg M. D., 2002, ApJ, 574, L43

Crause L. A. et al., 2016, Proc. SPIE, 9908, id. 990827 
Feast M. W., Whitelock P. A., 2000, MNRAS, 317, 460

Feast M. W., 2008, in First Middle EastAfrica, Regional IAU Meeting. p3 Online at http://www.mearim.cu.edu.eg/new/Proceeding.htm

Feast M. W., Whitelock P. A., Menzies J. W., 2006, MNRAS, 369,791

Feast M. W., Menzies J. W., Whitelock P. A., 2013, MNRAS, 428, L36

Green P., 2013, ApJ, 765, 12

Green P. J., Margon B., 1994, ApJ, 423, 723

Groenewegen M. A. T., Blommaert J. A. D. L., 2005, A\&A, 443, 143

Groenewegen M. A. T., de Jong T., Baas F., 1993, A\&AS, 101, 513

Haywood M., Di Matteo P., Snaith O., Calamida A., 2016, A\&A, 593, A82

Huxor, A. P., Grebel, E. K., 2015, MNRAS, 453, 2653

Hynes R. I. et al., 2014, ApJ, 780, 11

Ishihara D. et al., 2010, A\&A, 514, A1

Ishihara D., Kaneda H., Onaka T., Ita Y., Matsuura M., Matsunaga N., 2011, A\&A, 534, A79

Ita Y. et al., 2010, A\&A, 514, A2

Ita Y., Matsunaga N., 2011, MNRAS, 412, 2345

Izzard R. G., Jeffery C. S., Lattanzio J., 2007, A\&A, 470, 661

Lançon A., Wood P. R., 2000, A\&AS, 146, 217

Law D. R., Majewski S. R., 2016, ASSL, 420, 31

Libralato M. et al., 2015, MNRAS, 450, 1664

Loidl R., Lançon A., Jørgensen U. G., 2001, A\&A, 371, 1065

Marigo P., Girardi L., Bressan A., Groenewegen M. A. T., Silva L., Granato G. L., 2008, A\&A, 482, 883

McClure R. D., Woodsworth A. W., 1990, ApJ, 352, 709

McWilliam A. 2016, PASA, 33, e040

Menzies J. W., Feast M. W., Whitelock P. A., Matsunaga N., 2011, MNRAS, 414, 3492

Miszalski B., Mikołajewska J., Udalski A., 2013, MNRAS, 432, 3186

Mouhcine M., Lançon A., 2003, MNRAS, 338, 572

Nagashima, C. et al., 1999, in Nakamoto T., ed., Proc. Star Formation 1999. Nobeyama Radio Observatory, Nagano, p. 397

Nagayama T. et al., 2003, in Iye M., Moorwood A. F. M., eds, Proc. SPIE Vol. 4841, Instrument Design and Performance for Optical/Infrared Ground-based Telescopes. SPIE, Bellingham, p. 459

Nataf D. M., 2016a, PASA, 33, e023

Nataf D. M. et al., 2016, MNRAS, 456, 2692

Ness M., Freeman K., 2016, PASA, 33, 22

Ng Y. K., 1997, A\&A, 328, 211

Ng Y. K., 1998, A\&A, 338, 435

Nishiyama S. et al., 2006, ApJ, 638, 839

Ojha D. K., Tej A., Schultheis M., Omont A., Schuller F., 2007, MNRAS, 381, 1219

Pietrzyński G., et al., 2012, Nature, 484, 75

Plant K. A., Margon B., Guhathakurta P., Cunningham E. C., Toloba E., Munn J. A., 2016, ApJ, 833, 232

Recio-Blanco A. et al., 2017, A\&A, in press; arXiv:1702.04500

Renzini, A., Greggio, L., 1990, in Jarvis B. J., Terndrup D. M., eds, Bulges of Galaxies. ESO, Garching, p. 47

Skrutskie M. F. et al., 2006, AJ, 131, 1163

Soszyński I. et al., 2009, AcA, 59, 239

Soszyński I. et al., 2013, AcA, 63, 21

Totten E. J., Irwin M. J., Whitelock P. A., 2000, MNRAS, 314, 630

Tyson N. D., Rich R. M., 1991, ApJ, 367, 547

Udalski A. et al., 2002, AcA, 62, 133

Uttenthaler S., Blommaert J. A. D. L., Wood P. R., Lebzelter T., Aringer B., Schultheis M., Ryde N., 2015, MNRAS, 451, 1750 van Loon, J. Th. et al. 2003, MNRAS, 338, 857

Vassiliadis E., Wood P. R., 1993, ApJ, 413, 641
Whitelock P. A., 1993, IAUS, 153, 39

Whitelock P. A., Irwin M., Catchpole R. M., 1996, New Astron., 1,57

Whitelock P. A., Feast M. W., van Loon J. Th., Zijlstra A., 2003, MNRAS, 342, 86

Whitelock P. A., Feast M. W., Marang F., Groenewegen M. A. T., 2006, MNRAS, 369, 751

Wright N. J., Barlow M. J., Greimel R., Drew J. E., Matsuura M., Unruh Y. C., Zijlstra A. A., 2009, MNRAS, 400, 1413

Wood P. R., Habing H. J., McGregor P. J., 1998, A\&A, 336, 925 Wyckoff S., 1970, ApJ, 162, 203 